\title{
Dosimetry Determines the Initial OH Radical Concentration in Fast Photochemical Oxidation of Proteins (FPOP)
}

\author{
Ben Niu, Hao Zhang, Daryl Giblin, Don L. Rempel, Michael L. Gross \\ Department of Chemistry, Washington University in St. Louis, St. Louis, MO 63130-4899, USA
}

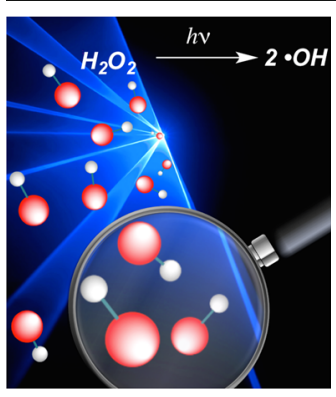

Abstract. Fast photochemical oxidation of proteins (FPOP) employs laser photolysis of hydrogen peroxide to give $\mathrm{OH}$ radicals that label amino acid side-chains of proteins on the microsecond time scale. A method for quantitation of hydroxyl radicals after laser photolysis is of importance to FPOP because it establishes a means to adjust the yield of $\bullet \mathrm{OH}$, offers the opportunity of tunable modifications, and provides a basis for kinetic measurements. The initial concentration of $\mathrm{OH}$ radicals has yet to be measured experimentally. We report here an approach using isotope dilution gas chromatography/mass spectrometry (GC/MS) to determine quantitatively the initial - $\mathrm{OH}$ concentration (we found $\sim 0.95 \mathrm{mM}$ from $15 \mathrm{mM} \mathrm{H}_{2} \mathrm{O}_{2}$ ) from laser photolysis and to investigate the quenching efficiencies for various $\bullet O H$ scavengers.

Keywords: FPOP, Dosimetry, Isotope Dilution, Gas Chromatography/Mass Spectrometry

Received: 10 December 2014/Revised: 22 January 2015/Accepted: 27 January 2015/Published Online: 25 February 2015

\section{Introduction}

$\mathrm{P}$ rotein footprinting examines protein structure and conformational changes by monitoring solvent accessibility and/ or H-bonding by using either modification or cleavage reactions [1]. The marriage of mass spectrometry (MS) proteomics methods and protein footprinting allows protein structure, function, dynamics, interactions with ligands, and stoichiometry to be examined [2]. Hydroxyl-radical oxidation is one method of "protein footprinting," a term coined by Chance et al. [3] for synchrotron-induced labeling. Hettich and coworkers [4] had earlier explored slow labeling via Fenton chemistry. Although there are other ways to produce $\bullet \mathrm{OH}[5-$ 7], the "fast photochemical oxidation of proteins" (FPOP) method shares the advantages that a stable, irreversible covalent modification is installed in nearly four-fifth of the aminoacid residues, providing higher coverage than most labeling methods. Given that $\cdot \mathrm{OH}$ is comparable in size to a water molecule, FPOP is a probe of solvent accessibility. Moreover, FPOP provides a fast "snapshot" of protein structure $(\sim 1 \mu \mathrm{s})$ without concern for rearrangements or loss of label during

Electronic supplementary material The online version of this article (doi:10.1007/s13361-015-1087-0) contains supplementary material, which is available to authorized users.

Correspondence to: Michael Gross; e-mail: mgross@wustl.edu subsequent sample handling and proteolysis, and it can accommodate other radical reagents [8,9].

FPOP utilizes a pulsed laser to photolyze hydrogen peroxide to generate two $\cdot \mathrm{OH}$ that rapidly modify proteins in a flow system [10]. The laser provides a spatially small, high flux of light, maximizing the exposure of small plugs of protein solution to radicals and ensuring all but a small fraction of the protein in the flow is irradiated only once [11]. FPOP is a practical approach, requiring only a modest laser and syringe pump [12-14].

Missing thus far in the development of FPOP is a method that gives the initial concentration of $\bullet \mathrm{OH}$, allowing rational means for tuning. The concentration of $\bullet \mathrm{OH}$ is also an input to any kinetic analysis to control the approach. Quantitation of -OH generated in FPOP is difficult because the radicals are short-lived. Hambly and Gross [10] estimated the initial concentration of $\cdot \mathrm{OH}$ to be $1 \mathrm{mM}$, consistent with the molar absorptivity of $\mathrm{H}_{2} \mathrm{O}_{2}$ and the quantum yield of $\bullet \mathrm{OH}$. Chen et al., using LC/MS, measured preliminarily the $[\bullet \mathrm{OH}]$ to be $0.42 \mathrm{mM}$ [15]. The high speed of oxidative modifications in FPOP, however, can be placed on a firmer ground by measuring the initial $[\bullet \mathrm{OH}]$ with a routine method.

Here we report a determination of the initial concentration of $\bullet \mathrm{OH}$ upon laser photolysis by using isotope dilution $\mathrm{GC} / \mathrm{MS}$, which is sensitive, specific [16, 17], and gives good accuracy, precision, and correction for analyte loss during handling [18]. We selected unlabeled phenylalanine as a "dosimeter 
molecule" to quantify the $\cdot \mathrm{OH}$ and $d_{5}$-phenylalanine as the internal standard. Phenylalanine is suitable because it is reactive towards $\bullet \mathrm{OH}$ (rate constant of $6.9 \times 10^{9} \mathrm{M}^{-1} \mathrm{~s}^{-1}[19]$ ), and it yields simple oxidation products by a mechanism discussed elsewhere [1].

We measured the initial $\bullet \mathrm{OH}$ concentration in FPOP and found that it is comparable to our previous estimate [10]. Further, we measured $[\cdot \mathrm{OH}]$ available to label proteins as a function of both the nature and concentration of the scavenger (i.e., methionine, histidine, and glutamine).

\section{Experimental}

\section{Materials and Reagents}

Unlabeled phenylalanine and $d_{5}$-phenylalanine were provided by Jan Crowley. $L$-glutamine, $L$-methionine, $L$-histidine, catalase, $30 \%$ hydrogen peroxide, $N, O$-bis(trimethylsilyl)fluoroacetamide (BSTFA), phosphate-buffer saline (PBS), and HPLC-grade solvents were from Sigma Aldrich (St. Louis, MO, USA).

\section{Calibration}

A series of solutions containing increasing amounts of $d_{0^{-}}$ phenylalanine $(0,10,25,50,100 \mathrm{nmol})$ were dissolved in PBS buffer (10 $\mathrm{mM}$ phosphate buffer, $138 \mathrm{mM} \mathrm{NaCl}$, and $2.7 \mathrm{mM} \mathrm{KCl}, \mathrm{pH} 7.4$ ), mixed with $\mathrm{H}_{2} \mathrm{O}_{2}$, and submitted to FPOP without pulsing the laser to correct for any analyte losses. Methionine and catalase were added to each vial immediately following to simulate FPOP conditions. A constant amount of $d_{5}$-phenylalanine internal standard (100 nmol) was then added to each vial.

After removing the solvent by speed-vac, the samples were treated with BSTFA to form trimethylsilyl derivatives that were analyzed by GC/high resolution MS with extracted ion chromatography and integration of ion peaks for the standard and unknown to give an intensity ratio of the analyte and the internal standard. The ratio was plotted as a function of the amount of $d_{0}$-phenylalanine initially added.

\section{FPOP Dosimetry Experiment}

Our goal was to determine the yield of $\bullet \mathrm{OH}$ when there was no scavenger in the system (Scheme 1) by incorporating the laser pulse for FPOP, as described previously [10]. $d_{0}$-Phenylalanine $(100 \mathrm{nmol})$ as dosimeter was dissolved in PBS buffer, and $\mathrm{H}_{2} \mathrm{O}_{2}$ was added just prior to syringe infusion; their concentrations were 2 and $15 \mathrm{mM}$, respectively. The sample solution was advanced at a rate of $23 \mu \mathrm{L} / \mathrm{min}$ by a syringe pump (Harvard Apparatus, Holliston, MA, USA). The excimer laser power (GAM Laser Inc., Orlando, FL, USA) at $248 \mathrm{~nm}$ was adjusted to $\sim 40 \mathrm{~mJ} /$ pulse at a frequency of $7.0 \mathrm{~Hz}$, affording an exclusion volume fraction of $20 \%$ (the volume not irradiated by laser, sandwiched between plugs of irradiated solution). All collections were in vials containing $500 \mathrm{nM}$ catalase and $70 \mathrm{mM}$ methionine, preventing further oxidation by $\mathrm{H}_{2} \mathrm{O}_{2}$. A solution containing $100 \mathrm{nmol}$ of $d_{5}$-phenylalanine was added to each vial, the solution dried on a speed vac, derivatized with BSTFA, and analyzed by GC/MS to give the amount of $d_{0}$-phenylalanine reacted, which equals the amount of $\bullet \mathrm{OH}$ generated.

\section{Gas Chromatography/Mass Spectrometry}

Samples were diluted 10:1 before injecting into an Agilent 7200 Q-TOF GC/MS (Santa Clara, CA, USA) with a split of 10:1, to avoid column overload and detector saturation. The inlet temperature was $280^{\circ} \mathrm{C}$. The gas chromatograph was an Agilent 7890 equipped with an Agilent 19091S HP-5 ms col-

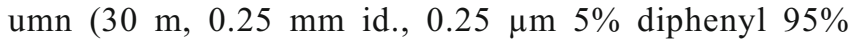
dimethylpolysiloxane film coating). The GC oven temperature was at $80{ }^{\circ} \mathrm{C}$ for $2 \mathrm{~min}$ after sample auto-injection and then ramped at a rate of $10^{\circ} \mathrm{C} / \mathrm{min}$ to a final temperature of $300{ }^{\circ} \mathrm{C}$, which was held for $6 \mathrm{~min}$. All spectra were acquired in the positive-ion EI mode.

\section{Results and Discussion}

To measure the $[\bullet \mathrm{OH}]$, we derivatized the carboxyl and amine groups of phenylalanine with trimethylsilyl groups, taking

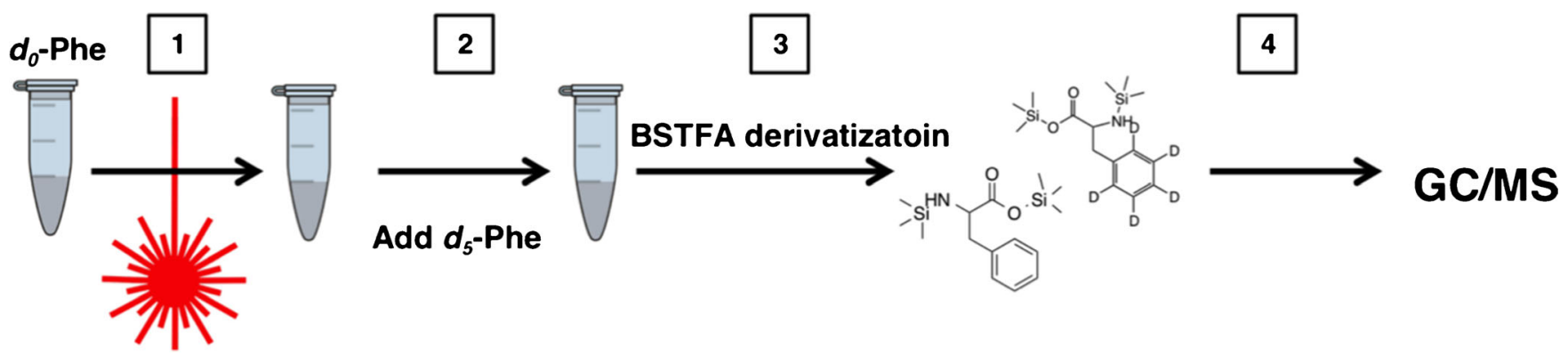

Scheme 1. (1) Without scavenger, $100 \mathrm{nmol}$ of dosimeter ( $d_{0}$-phenylalanine) was subjected to FPOP, generating tyrosine-like products from $\bullet \mathrm{OH}$ attack on the aromatic ring. (2) Internal standard ( $d_{5}$-phenylalanine) added to vial. (3) BSTFA added for derivatization of both dosimeter and internal standard. (4) Products analyzed by GC/MS 
advantage of this well-known protocol [20]. We chose fragment ions of $\mathrm{m} / \mathrm{z} 294.1340$ and 299.1654 (derivatized $d_{0}$-phenylalanine and $d_{5}$-phenylalanine) because the molecular ions $\left(\mathrm{M}^{+\bullet}\right.$ ) of $\mathrm{m} / \mathrm{z} 309.1575$ and 314.1889 ions were not detectable [21] (structures and EI mass spectra are in SI). Other appropriate fragment ions are of $\mathrm{m} / \mathrm{z} 266.1391$ and 271.1705; and of $\mathrm{m} / \mathrm{z}$ 91.0542 and 96.0856.

To conduct the analysis, we held constant the amount of $d_{5^{-}}$ phenylalanine derivative in each sample (100 nmol), so there was little change in its ion-current peak integral (see SI Figure 2), and increased $d_{0}$-phenylalanine from 0 to $100 \mathrm{nmol}$. The ratio of the ion-current peak integrals $\left(d_{0} / d_{5}\right)$ was obtained from extracted ion chromatograms and plotted as a function of amount of added $d_{0}$-phenylalanine. The ions of $\mathrm{m} / \mathrm{z} 294.1340$ and 299.1654 gave a ratio of peak integrals $\left(d_{0} / d_{5}\right)$ as a linear function of the amount of $d_{0}$-phenylalanine over 0-100 nmol (see SI Figure 3). The curve (slope of 0.0061, y-axis intercept of 0.015 , and an $\mathrm{R}^{2}$ of 0.9970 ) provided the interpolated concentration of remaining $d_{0}$-phenylalanine. By subtracting this amount of $d_{0}$-phenylalanine from the initial amount, we obtained the amount of consumed $d_{0}$-phenylalanine, which is the amount of $\bullet \mathrm{OH}$ from each laser pulse that reacted with the dosimeter. From these experiments, the $\bullet \mathrm{OH}$ concentration that reacted with the dosimeter from each laser pulse was 0.67 $\pm 0.08 \mathrm{mM}$ from a starting solution of $15 \mathrm{mM} \mathrm{H}_{2} \mathrm{O}_{2}$, an amount close to our earlier estimate [10].

We selected two other fragment-ion pairs $(\mathrm{m} / z 266.1391$ and 271.1705; and $\mathrm{m} / \mathrm{z} 91.0542$ and 96.0856) to build two other calibrations (see SI Figure 3) that afforded measured $[\cdot \mathrm{OH}]$ of $0.69 \pm 0.12$ and $0.68 \pm 0.12 \mathrm{mM}$, respectively. Two control experiments were included, one without laser and the other without $\mathrm{H}_{2} \mathrm{O}_{2}$.

The initial $[\bullet \mathrm{OH}]$ should be slightly higher because the measured value is reduced by $\bullet \mathrm{OH}$ self-recombination, which competes with the reaction of $\bullet \mathrm{OH}$ and dosimeter. We simulated by kinetic modeling the amount of phenylalanine product to predict an initial $[\bullet \mathrm{OH}]$. A search varied the postulated initial $[\bullet \mathrm{OH}]$ until the calculated $\bullet \mathrm{OH}$ concentration that reacted with the dosimeter matched the experimentally determined value $(0.67 \mathrm{mM})$. The best fit showed the initial $[\bullet \mathrm{OH}]$ to be $0.95 \mathrm{mM}$. The simulations also included the Haber-Weiss chain reaction, which has little effect. We did not model, however, any reaction of $\bullet \mathrm{OOH}$ produced in the final step of phenylalanine modification [1].

\section{Effect of Scavenger}

In a typical FPOP experiment, scavengers limit the available $[\cdot \mathrm{OH}]$ for footprinting. Thus, it is of interest to measure the $[\bullet \mathrm{OH}]$ when using scavengers of different nature or concentrations, facilitating creation of an improved FPOP platform with scavenger-tunable $[\bullet \mathrm{OH}]$. Tuning the $[\bullet \mathrm{OH}]$ is important because different proteins usually require different $[\cdot \mathrm{OH}]$ to achieve modifications that are sufficient to reveal structural information, yet do not give overoxidation. Moreover, varying the scavenger provides the means for measuring the kinetics of

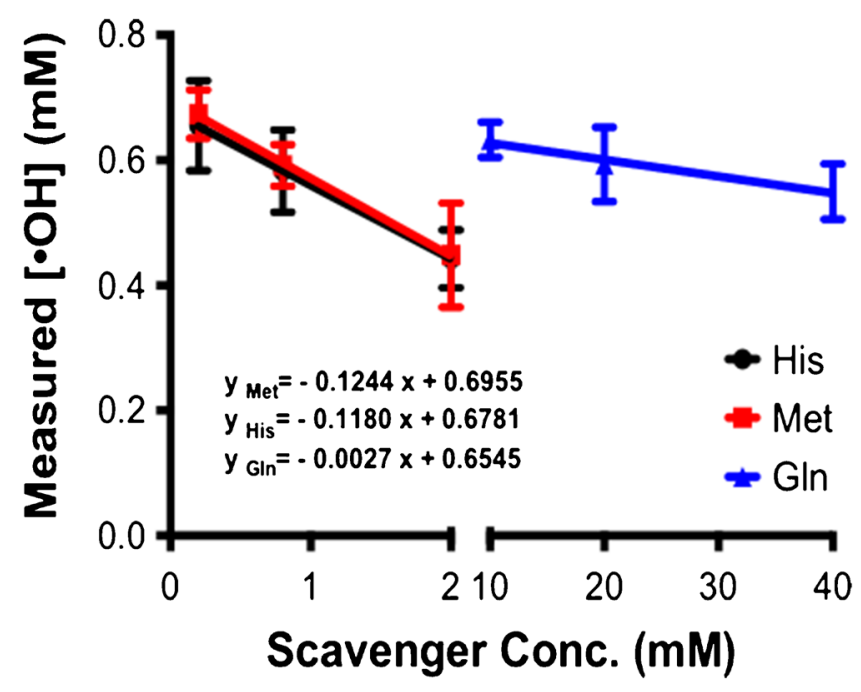

Figure 1. Dose-response curves determined from fragment ions of $\mathrm{m} / \mathrm{z} 294.1340$ and 299.1654 for Met, His, and Gln. See SI for dose-response curves determined from other fragment ions (i.e., $\mathrm{m} / \mathrm{z} 266.1391$ and 271.1705; and $\mathrm{m} / \mathrm{z} 91.0542$ and 96.0856). Note that the lifetime of the radicals is $\sim 1 \mu \mathrm{s}$ when glutamine is $20 \mathrm{mM}$-see Fig. 1 .

radical modification reactions. To test this, we used different concentrations of glutamine (10,20,40 mM) as scavenger and measured $[\cdot \mathrm{OH}]$ to establish a relationship between measured $[\bullet \mathrm{OH}]$ and scavenger concentration. Furthermore, histidine and methionine were also tested as a function of concentration $(0.2$, $0.8,2.0 \mathrm{mM}$ ) (Figure 1). For each scavenger (His, Met, Gln), the measured $[\bullet \mathrm{OH}]$ decreases as scavenger concentration increases. Moreover, extrapolation of the curves to the y-axis, gives approximately the same intercept, $[\bullet \mathrm{OH}]$ with no scavenger (i.e., $0.67 \mathrm{mM}$ ) (see Figure 1 and SI Figure 4). The equation for each scavenger response curve is also given in those figures. The ratios of the rate constants of glutamine,

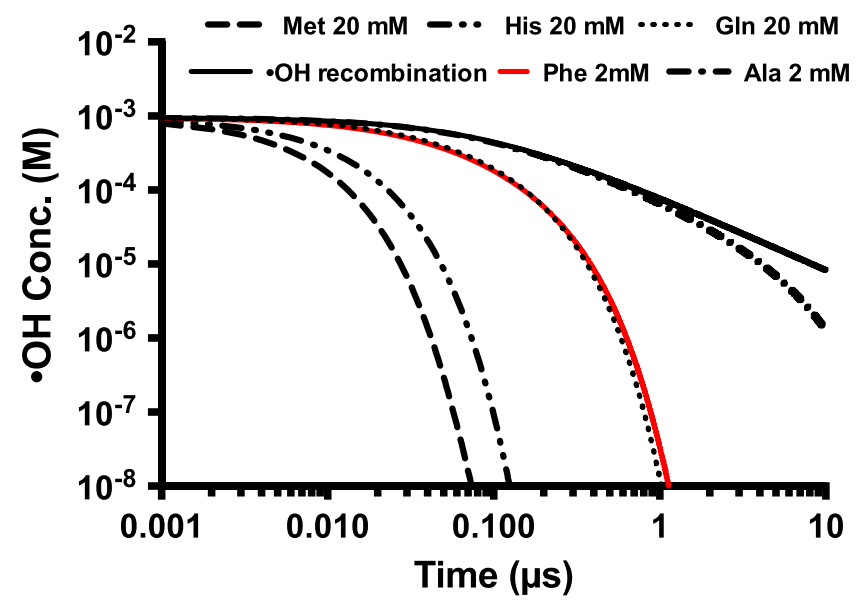

Figure 2. Numerical simulation of $\bullet \mathrm{OH}$ concentration versus time. The simulation includes the $\bullet \mathrm{OH}$ reaction with scavengers, the recombination of $\bullet \mathrm{OH}$, and the Haber-Weiss chain reaction. Lifetime of $\bullet \mathrm{OH}(\sim 100$ ns to more than $10 \mu \mathrm{s})$ depends on the nature and concentration of the scavenger 
histidine, and methionine towards $\bullet \mathrm{OH}\left(5.4 \times 10^{8} \mathrm{M}^{-1} \mathrm{~s}^{-1}\right.$, $4.8 \times 10^{9} \mathrm{M}^{-1} \mathrm{~s}^{-1}$, and $8.5 \times 10^{9} \mathrm{M}^{-1} \mathrm{~s}^{-1}$, respectively [1]) should predict the ratios of the line slopes, and this is approximately correct. Discrepancies may be due to differences in our methods and conditions compared with the original kinetic studies.

For different amino-acid scavengers, the rate constants differ by three orders of magnitude [1]. Taking advantage of this, we can adjust the $[\bullet \mathrm{OH}]$ available for footprinting by varying either the nature or concentration of the scavenger. The outcomes may be significant because the time range available to FPOP can be shifted from either $\sim 1 \mu$ s to $\sim 100$ ns (scavengers of higher reactivity or concentration), or to near ms (scavengers of lower reactivity or concentration). A numerical simulation of $[\cdot \mathrm{OH}]$ by using different scavengers (Figure 2 ) shows that the lifetime is approximately $1 \mu$ s when using $20 \mathrm{mM}$ glutamine as scavenger but adjustable to $\sim 0.1 \mu$ s by using methionine or histidine or to $100 \mu$ s with alanine as scavengers (we assign the lifetime of the radicals to the time at which their concentrations are 100 times less than that of the protein). Adjusting the lifetime of $\bullet \mathrm{OH}$ enables labeling over a wider time scale, making FPOP a flexible tool to investigate various folding/ unfolding events. For example, some proteins fold to a native state by first forming secondary and then tertiary structure [22], whereas other proteins go through hydrophobic collapse and then form tertiary structure [23]. The time-course changes for FPOP are in the time range for the major steps of protein folding, allowing future studies on its details.

\section{Conclusion}

The specificity, sensitivity, and precision of isotope dilution GC/MS to identify and quantify the dosimeter recommend it for quantifying the initial $\bullet \mathrm{OH}$ concentration in FPOP. The approach permits optimization of FPOP conditions and adjustment of experimental parameters to realize tunable extents of modifications. Our next step is to study the effect of tuning, aiming for secure "hard" kinetic data that will allow comparison of results and lab-to-lab.

\section{Acknowledgments}

This research was supported by National Institute of General Medical Sciences (8 P41 GM103422-37 and 1R01GM108785) of the National Institute of Health. The authors thank Jan R. Crowley and Kwasi Mawuenyega for their generous advice and help.

\section{References}

1. Xu, G., Chance, M.R.: Hydroxyl radical-mediated modification of proteins as probes for structural proteomics. Chem. Rev. 107(8), 3514-3543 (2007)
2. Aebersold, R., Mann, M.: Mass spectrometry-based proteomics. Nature 422(6928), 198-207 (2003)

3. Maleknia, S.D., Brenowitz, M., Chance, M.R.: Millisecond radiolytic modification of peptides by synchrotron X-rays identified by mass spectrometry. Anal. Chem. 71(18), 3965-3973 (1999)

4. Sharp, J.S., Becker, J.M., Hettich, R.L.: Protein surface mapping by chemical oxidation: structural analysis by mass spectrometry. Anal. Biochem. 313(2), 216-225 (2003)

5. Xu, G., Chance, M.R.: Radiolytic modification of acidic amino acid residues in peptides: probes for examining protein-protein interactions. Anal. Chem. 76(5), 1213-1221 (2004)

6. Sharp, J.S., Becker, J.M., Hettich, R.L.: Analysis of protein solvent accessible surfaces by photochemical oxidation and mass spectrometry. Anal. Chem. 76(3), 672-683 (2004)

7. Fenton, H.J.H.: Oxidation of tartaric acid in presence of iron. J. Chem. Soc. 65, 899-910 (1894)

8. Gau, B.C., Chen, H., Zhang, Y., Gross, M.L.: Sulfate radical anion as a new reagent for fast photochemical oxidation of proteins. Anal. Chem. 82(18), $7821-7827$ (2010)

9. Chen, J., Cui, W., Giblin, D., Gross, M.L.: New protein footprinting: fast photochemical iodination combined with top-down and bottom-up mass spectrometry. J. Am. Soc. Mass Spectrom. 23(8), 1306-1318 (2012)

10. Hambly, D.M., Gross, M.L.: Laser flash photolysis of hydrogen peroxide to oxidize protein solvent-accessible residues on the microsecond timescale. J. Am. Soc. Mass Spectrom. 16(12), 2057-2063 (2005)

11. Gau, B.C., Sharp, J.S., Rempel, D.L., Gross, M.L.: Fast photochemical oxidation of protein footprints faster than protein unfolding. Anal. Chem. 81(16), 6563-6571 (2009)

12. Zhang, H., Gau, B.C., Jones, L.M., Vidavsky, I., Gross, M.L.: Fast photochemical oxidation of proteins for comparing structures of protein-ligand complexes: the calmodulin-peptide model system. Anal. Chem. 83(1), 311-318 (2011)

13. Jones, L.M., Sperry, J.B., Carroll, J.A., Gross, M.L.: Fast photochemical oxidation of proteins for epitope mapping. Anal. Chem. 83(20), 7657-7661 (2011)

14. Chen, J., Rempel, D.L., Gau, B.C., Gross, M.L.: Fast photochemical oxidation of proteins and mass spectrometry follow submillisecond protein folding at the amino-acid level. J. Am. Chem. Soc. 134(45), 18724-18731 (2012)

15. Chen, J.: Mass Spectrometry for determination of conformation and dynamics of proteins and structure and biosynthesis of bacterial peptidoglycan. Doctoral thesis, Washington University in St. Louis (December 2011)

16. Pomerantz, S.C., McCloskey, J.A.: Analysis of RNA hydrolyzates by liquid chromatography-mass spectrometry. Methods Enzymol. 193, 796$824(1990)$

17. Heinecke, J.W., Hsu, F.F., Crowley, J.R., Hazen, S.L., Leeuwenburgh, C., Mueller, D.M., Rasmussen, J.E., Turk, J.: Detecting oxidative modification of biomolecules with isotope dilution mass spectrometry: sensitive and quantitative assays for oxidized amino acids in proteins and tissues. Methods Enzymol. 300, 124-144 (1999)

18. Chapman, J.R.: Practical organic mass spectrometry. A Wiley-Interscience publication. Wiley, Chichester West Sussex, England (1985)

19. Masuda, T., Nakano, S., Kondo, M.: Rate constants for the reactions of OH radicals with the enzyme proteins as determined by the pnitrosodimethylaniline method. J. Radiat. Res. 14(4), 339-345 (1973)

20. Crowley, J.R., Yarasheski, K., Leeuwenburgh, C., Turk, J., Heinecke, J.W.: Isotope dilution mass spectrometric quantification of 3-nitrotyrosine in proteins and tissues is facilitated by reduction to 3-aminotyrosine. Anal. Biochem. 259(1), 127-135 (1998)

21. Watson, J.T., Sparkman, O.D.: Introduction to Mass Spectrometry: Instrumentation, Applications, and Strategies for Data Interpretation, 4th ed. John Wiley and Sons, Chichester, England; Hoboken, NJ (2007)

22. Gilmanshin, R., Williams, S., Callender, R.H., Woodruff, W.H., Dyer, R.B.: Fast events in protein folding: relaxation dynamics and structure of the I form of apomyoglobin. Biochemistry 36(48), 15006-15012 (1997)

23. Arai, M., Kondrashkina, E., Kayatekin, C., Matthews, C.R., Iwakura, M., Bilsel, O.: Microsecond hydrophobic collapse in the folding of Escherichia coli dihydrofolate reductase, an alpha/beta-type protein. J. Mol. Biol. 368(1), 219-229 (2007) 